\title{
EXOMOL: MOLECULAR LINE LISTS FOR EXOPLANET AND OTHER ATMOSPHERES
}

\author{
S.N. Yurchenko ${ }^{1}$ and J. Tennyson ${ }^{1}$
}

\begin{abstract}
This contribution lays out the scientific foundations of the project ExoMol. This project will produce molecular line lists for all molecules considered to be important for the modelling atmospheres of exoplanets and cool stars, which are prerequisites for the spectroscopic characterization of the atmospheres of these astrophysical objects. Towards this end 25 species are identified as key ones for meeting current demands for atmospheric models of exoplanets and brown dwarfs, impeded by the lack of fundamental data on the absorption of these species. The production of comprehensive and very large rotationvibration and rotation-vibration-electronic line lists requires a mixture of first principles quantum mechanical methods and empirical tuning based on laboratory spectroscopic data. ExoMol will rely on these methodologies and make extensive use of state-of-the-art computing.
\end{abstract}

\section{Introduction}

Since the discovery of the first extra-solar planet, the number of confirmed and candidate exoplanets has grown hugely. Focus has therefore shifted towards characterising these bodies and, in particular, determining what they are made of. Most astronomical discoveries rely on the use of fundamental atomic and molecular data. Thus interpreting observations of and modelling the atmospheres of planetary and stellar objects which can give chemical compositions and temperatures relies heavily on advances in laboratory astrophysics, especially in molecular spectroscopy. Despite considerable progress in computational spectroscopy over the last 10 years that has led to accurate and comprehensive description of some three-atomic molecules (water, $\mathrm{H}_{3}^{+}, \mathrm{HCN} / \mathrm{HNC}$ ), information on other important absorbers is still incomplete, particularly at temperatures significantly above room temperature. The overall aim of the ExoMol project is to provide accurate and detailed theoretical data on the spectroscopy of key constituents of the atmospheres of different astrophysical bodies such as cool stars, exoplanets and planetary discs.

1 Department of Physics and Astronomy, University College London, Gower Street, WC1E 6BT London, UK 
Such data are most conveniently organized in the form of catalogues of transitions, so-called line lists. ExoMol will generate line lists for all molecules likely to be important in the atmospheres of extra-solar planets and cool stars. The identification of water in an exoplanet by Tinetti et al. (2007) based on the state-of-the-art water BT2 (Barber et al. 2006) line list is an example demonstrating the potential of reliable theoretical line lists.

Accurate modelling of the atmospheres of planetary and stellar objects is still impeded by the lack of spectroscopic data on the main absorbers. Consequently, there are still no data available for many of the important constituents of the atmospheres of exoplanets and brown dwarfs. Information on other absorbers is either inaccurate or incomplete and thus requires substantial improvement. For example, the recent methane line list of Warmbier et al. (2009) offers neither the accuracy nor the completeness required for models of hot methane spectra found in exoplanets. Furthermore, the growing number of the astrophysical surveys will create an increasing demand for efficient spectroscopy of the hundreds to thousands of objects observed per square degree. Existing molecular line lists are inadequate for the anticipated level of sensitivity required to interpret these observations. ExoMol will develop computational tools and generate more complete and accurate data to respond to these demands.

There are likely to be demands for line lists of hydrogenated species such as $\mathrm{H}_{2} \mathrm{~S}, \mathrm{PH}_{3}$, acetylene, ethane and propane. Acetylene belongs to the precursors to DNA and protein and is considered to be particular important for carbon stars. The phosphine is of special importance for the atmosphere spectroscopy of Jupiter and Saturn. $\mathrm{H}_{2} \mathrm{~S}$ is expected to be an important constituent of some exoplanets. Nitric acid is also a possible bio-signature molecule. The demands for the line list of $\mathrm{SO}_{2}$ is driven by theoretical models of solar system planetary atmospheres, where it is a known constituent. From an astrophysical perspective, the absence of satisfactory line list of methane means that it is not possible to model accurately the atmospheres of late brown dwarfs or gas giant classes of exoplanets. The amount of this species present also remains completely uncertain because the necessary laboratory data on their hot spectra is simply not available.

The goal of the project is to establish systematic production of line lists for all molecules likely to be observable in atmospheres of exoplanets and cool stars in the foreseeable future. In Table 1 we summarise our selection of the molecules based on current demands for models of exoplanets and brown dwarfs, impeded by the lack of fundamental data on the absorption of these species. However these demands may change over the course of the project since the characterisation of exoplanets is a rapidly developing field.

The data produced will have a wide range of applications also far beyond the direct scope of ExoMol, reflecting the interdisciplinary and intersectional character of the project:

- The generated line lists will be essential for modelling planetary and stellar atmospheres, including hot gas giant planets (e.g. "super- Jupiters") and cool stars (far the most common in the local galaxy). 
Table 1. Exomol list of molecules: Molecular line lists available and planned, categorised by chemistry.

\begin{tabular}{|c|c|c|c|}
\hline & $\begin{array}{l}\text { Primordial (Metal- } \\
\text { poor) }\end{array}$ & $\begin{array}{l}\text { Terrestrial Planets } \\
\text { (Oxidising) }\end{array}$ & 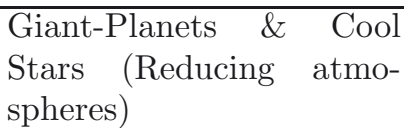 \\
\hline $\begin{array}{l}\text { Already } \\
\text { available }\end{array}$ & $\begin{array}{l}\mathrm{H}_{2}, \quad \mathrm{HD}^{+}, \quad \mathrm{LiH}, \\
\mathrm{LIH}^{+}, \mathrm{HeH}^{+}, \mathrm{H}_{3}^{+}, \\
\mathrm{H}_{2} \mathrm{D}^{+}\end{array}$ & $\begin{array}{l}\mathrm{OH}, \mathrm{CO}_{2}, \mathrm{O}_{3}, \mathrm{NO}, \\
\mathrm{H}_{2} \mathrm{O}, \mathrm{NH}_{3}\end{array}$ & $\begin{array}{l}\mathrm{H}_{2}, \mathrm{CN}, \mathrm{CH}, \mathrm{CO}, \mathrm{CO}_{2}, \\
\mathrm{TiO}, \mathrm{HCN} / \mathrm{HNC}, \mathrm{H}_{2} \mathrm{O}, \\
\mathrm{NH}_{3}\end{array}$ \\
\hline ExoMol & $\mathrm{BeH}$ & $\begin{array}{l}\mathrm{O}_{2}, \quad \mathrm{CH}_{4}, \quad \mathrm{SO}_{2}, \\
\mathrm{HOOH}, \\
\mathrm{HNO}_{3} \mathrm{CO},\end{array}$ & $\begin{array}{l}\mathrm{CH}_{4}, \mathrm{PH}_{3}, \quad \mathrm{C}_{2}, \quad \mathrm{C}_{3}, \\
\mathrm{HCCH}, \mathrm{C}_{2} \mathrm{H}_{6}, \mathrm{C}_{3} \mathrm{H}_{8}, \mathrm{VO}, \\
\mathrm{O}_{2}, \quad \mathrm{AlO}, \quad \mathrm{MgO}, \quad \mathrm{BeH}, \\
\mathrm{CrH}, \mathrm{MgH}, \mathrm{FeH}, \mathrm{CaH}, \\
\mathrm{AlH}, \mathrm{SiH}, \mathrm{NiH}, \mathrm{TiH}, \mathrm{YO}\end{array}$ \\
\hline
\end{tabular}

- Accurate line lists are of significant importance for assignment of laboratory spectra. The advantages of the synthetic spectroscopic information are (i) high predictive power, (ii) data completeness, (iii) reliable intensity information and (iv) access to the temperature regimes which are not always accessible experimentally.

- The opacities of the most species participating in atmospheric processes (on Earth, exoplanets or cool stars) are still incomplete. Molecular line list data are essential for solving the opacity problem.

- The generated molecular line lists will contribute directly to improving atmospheric databases, such as HITRAN and GEISA. In particular the representations of ammonia and methane in these databases require substantial improvement.

- The use of molecular line lists (especially of water and methane) for modelling combustion can lead to much more accurate flame temperatures, which is important for studies of gas turbine engines.

- Comprehensive information on rotation-vibration energies is necessary for calculation of partition functions and hence specific heats and other thermodynamic properties.

The planned EChO mission (Tessenyi et al. 2012; Tinetti et al. 2012) will study the atmospheres of exoplanets using molecular spectroscopy. One of the objectives of this mission is characterization of the conditions on exoplanets potentially suitable for generating and sustaining life ("super-Earths"). Thus EChO may become a big consumer of the data produced by ExoMol.

The generated line lists will be made publically available at www.exomol .com. This site will also provide tools for generating absorption/emmision spectra, partition functions, specific heats and other thermodynamic properties for a wide 
range of temperatures. Finally, the line lists of relevant systems will be applied for modelling the atmospheres of (exo-)planets and cool stars. Such lists of states and transitions will be suitable for straightforward integration in existing models of stellar atmospheres, to estimate their effect on radiation transport, their physical structure and the resulting spectra. The spectra of exoplanets and brown dwarfs made up of many molecular and atomic absorbers are extremely rich in structure, each with hundreds of thousands to millions of spectral lines. Their spectra can therefore be used as a tool to determine fundamental parameters of the atmospheres of planetary and stellar objects, such as the effective temperature.

\section{Methods}

In order to simulate molecular spectra, absorption or emission, information on the (ro-vibrational) energy levels as well as on the molecular absorbance is required. Towards this end one needs to solve as accurately as possible the Schrödinger equation for the nuclear motion. For most of the molecules listed in Table 1 only vibration-rotation transitions within the ground electronic state are necessary to consider. However some molecules undergo electronic transitions at near infrared or visible wavelengths and hence excited electronic states will be important.

Within the Born-Oppenheimer approximation, the calculation of line lists of rotation-vibration transitions for a stable polyatomic molecule consists of the following steps: (a) accurate $a b$ initio calculations to create a potential energy surface (PES) and a dipole moment surface (DMS); (b) nuclear motion calculations to provide energy levels and wave functions; (c) calculation of transition dipole moments using the wave functions and dipole moment surfaces.

Even an accurate $a b$ initio PES cannot deliver ro-vibrational energies with sufficiently high accuracy and therefore has to be empirically refined by least-squares fitting to experimental energies or frequencies to give a "spectroscopic" potential; such potentials can provide theoretical line positions with near-experimental accuracy. However an ab initio DMS computed at a high level of theory (such as coupled clusters $\operatorname{CCSD}(\mathrm{T})$ method with a large basis) has the potential to provide a very reasonable description of the intensities. Moreover, properly obtained $a b$ initio intensities in all but few cases are superior to data provided by experiment.

Treatment of the nuclear motion problem for diatomics is relatively straightforward. For uncoupled electronic states, we will simply use the program LEVEL by Le Roy (2007). In more complicated cases it will be necessary to consider the coupling between different electronic states explicitly in the calculation. Work on diatomic line lists is actively underway with line lists for the $X^{2} \Sigma^{+}$states of $\mathrm{BeH}$, $\mathrm{MgH}$ and $\mathrm{CaH}$ (Yadin et al. 2012).

The rotation-vibration Schrödinger equation for a polyatomic molecule is solved most commonly by variational methods, where the truncated matrix representation of the rotation-vibration Hamiltonian is constructed and diagonalized in terms of a suitable basis set. We will employ variational programs like DVR3D (Tennyson et al. 2004), TROVE (Yurchenko et al. 2007), and WAVR4 (Kozin et al. 2004) to calculate the rotation-vibration energies, eigenfunctions, and matrix elements of 
the electric dipole moment of the molecules in question, quantities necessary for simulation of spectra.

For large molecules, the dimension of the matrix blocks to be diagonalized increases very rapidly with rotational excitation, and even at low rotational excitation these matrix blocks may be so large that numerical diagonalization is no longer practical. In the representation of the symmetrized basis functions the Hamiltonian matrix becomes block-diagonal according to the corresponding irreducible representations. This simplifies the calculations of the energies and wavefunctions and enforces the symmetry selection rules for the calculated transitions, mandatory for intensity simulations.

For the intensity simulations, line strengths, Einstein coefficients, and integrated absorption coefficient will be computed for individual rotation-vibration transitions at different temperatures as described in by Yurchenko et al. (2011). The BYTe line list for ammonia presented in this work is an example of a line list production based on the presented scheme. This line list is a response to the growing astrophysical demands for information on the absorption properties of ammonia for a broad range of temperatures. It was already included in several astrophysical studies (Beaulieu et al. 2010; Lucas et al. 2010). The accuracy of theoretical transition intensities is confirmed by experiment. This in conjunction with the comprehensive character of the theoretical line list data reflects the extreme importance of our project.

\section{Conclusion}

To conclude, the overall aim of ExoMol is to establish systematic production of line lists for all molecules likely to be important in the atmospheres of extra-solar planets and cool stars. ExoMol will deliver molecular line lists, i.e. catalogues of rotation-vibration transition composed from energies, assignments, frequencies, Einstein coefficients, integrated absorption coefficients for a number of molecules of atmospheric and astrophysical importance. These line lists, and other information about the project, will also be made available via the ExoMol website, www.exomol.com. A more detailed description of the aims, scope and methodology of the project can be found in the article Tennyson \& Yurchenko (2012).

This work is supported by ERC Advanced Investigator Project 267219. We thank those astronomers who have shared their insight with us in the formulation of this project and in particular Giovanna Tinetti, Peter Bernath, Caitlin Griffiths, Yakiv Pavlenko and Mark Swain. We also thank the present members of the ExoMol team, Ala'a Azzam, Bob Barber, Christian Hill, Lorenzo Lodi, Andrei Patrascu, Oleg Polyansky and Clara Sousa-Silva, as well as Attila Császár and Andrew Kerridge and for many helpful discussions on the project.

\section{References}

Barber, R.J., Tennyson, J., Harris, G.J., \& Tolchenov, R.N., 2006, MNRAS, 368, 1087

Beaulieu, J.P., Kipping, D.M., Batista, V., et al., 2010, MNRAS, 409, 963 
Kozin, I.N., Law, M.M., Tennyson, J., \& Hutson, J.M., 2004, Comput. Phys. Commun., 163, 117

Le Roy, R.J., 2007, LEVEL 8.0 A Computer Program for Solving the Radial Schrödinger

Equation for Bound and Quasibound Levels, University of Waterloo Chemical Physics

Research Report CP-663, http://leroy.uwaterloo.ca/programs/

Lucas, P.W., Tinney, C.G., Burningham, B., et al., 2010, MNRAS, 408, L56

Tennyson, J., Kostin, M.A., Barletta, P., et al., 2004, Comput. Phys. Commun., 163, 85

Tennyson, J., \& Yurchenko, S.N., 2012, MNRAS, 425, 21

Tessenyi, M., Ollivier, M., Tinetti, G., et al., 2012, ApJ, 746, 45

Tinetti, G., et al., 2012, Experimental Astronomy, 34, 311

Tinetti, G., Vidal-Madjar, A., Liang, M.-C., et al., 2007, Nature, 448, 169

Warmbier, R., Schneider, R., Sharma, A. R., et al., 2009, A\&A, 495, 655

Yadin, B., Vaness, T., Conti, P., et al., 2012, MNRAS, 425, 34

Yurchenko, S.N., Barber, R.J., \& Tennyson, J., 2011, MNRAS, 413, 1828

Yurchenko, S.N., Thiel, W., \& Jensen, P., 2007, J. Mol. Spectrosc., 245, 126 